\title{
MULTIPLE SOLUTIONS FOR HARDY NONLOCAL FRACTIONAL ELLIPTIC EQUATIONS IN $\mathbb{R}^{N}$
}

\author{
SHAHLA AMIRI ${ }^{1}$, NEMAT NYAMORADI $^{2, *}$, ABOLFAZL BEHZADI $^{1}$ \\ ${ }^{1}$ Department of Mathematics, Faculty of Mathematical Sciences, University of Mazandaran, Babolsar, Iran \\ ${ }^{2}$ Department of Mathematics, Faculty of Sciences, Razi University, 67149 Kermanshah, Iran
}

\begin{abstract}
In this paper, we use Ekeland's variational principle to study the existence of at least three nontrivial solutions for the following critical nonlocal fractional Hardy elliptic equation $(-\Delta)^{s} u-\gamma \frac{u}{|x|^{2 s}}=\frac{|u|^{2 *}(\alpha)-2 u}{|x|^{\alpha}}+\lambda f(x, u)$ in $\mathbb{R}^{N}$, where $N>2 s$, $0<s<1, \gamma, \lambda$ are real parameters, $2_{s}^{*}(\alpha)=\frac{2(N-\alpha)}{N-2 s}$ is critical Hardy-Sobolev exponent with $\alpha \in[0,2 s), f: \mathbb{R}^{N} \times \mathbb{R} \rightarrow \mathbb{R}$ is a suitable function and $(-\Delta)^{s}$ is the fractional Laplace operator.
\end{abstract}

Keywords. Fractional Laplacian; Solution; Hardy-Sobolev exponent; Ekeland's variational principle.

2010 Mathematics Subject Classification. 35R11, 35A15, 35J60, 35J20.

\section{INTRODUCTION}

In this paper, we investigate the existence and multiplicity of solutions for the following critical nonlocal fractional Hardy elliptic equation

$$
(-\Delta)^{s} u-\gamma \frac{u}{|x|^{2 s}}=\frac{|u|^{2 *}(\alpha)-2 u}{|x|^{\alpha}}+\lambda f(x, u), \text { in } \mathbb{R}^{N},
$$

where $N>2 s, 0<s<1, \gamma, \lambda$ are real parameters, $2_{s}^{*}(\alpha)=\frac{2(N-\alpha)}{N-2 s}$ is critical Hardy-Sobolev exponent with $\alpha \in[0,2 s)$ and $f: \mathbb{R}^{N} \times \mathbb{R} \rightarrow \mathbb{R}$ is a suitable function and $(-\Delta)^{s}$ is the fractional Laplace operator, which, up to normalization factors, may be defined as

$$
(-\Delta)^{s} u(x)=-\frac{1}{2} \int_{\mathbb{R}^{N}} \frac{u(x+y)+u(x-y)-2 u(x)}{|x-y|^{N+2 s}} d y
$$

${ }^{*}$ Corresponding author.

E-mail addresses: sh.amiri@stu.umz.ac.ir (S. Amiri), nyamoradi@ razi.ac.ir; neamat80@yahoo.com (N. Nyamoradi), behzadi@umz.ac.ir (A. Behzadi).

Received December 5, 2018; Accepted May 3, 2019.

(C)2019 Journal of Nonlinear Functional Analysis 
for $x \in R^{N}$. Moreover, Let $£$ be the Schwartz space of rapidly decaying $C^{\infty}$ functions in $R^{N}$. Then, for any $u \in £$ and $s \in(0,1),(-\Delta)^{s}$ is defined as

$$
\begin{aligned}
(-\Delta)^{s} u(x) & =C(N, s) P . V \cdot \int_{\mathbb{R}^{N}} \frac{u(x)-u(y)}{|x-y|^{N+2 s}} d y \\
& =C(N, s) \lim _{\varepsilon \rightarrow 0} \int_{f B_{\varepsilon}(x)} \frac{u(x)-u(y)}{|x-y|^{N+2 s}} d y,
\end{aligned}
$$

where $£ B_{\varepsilon}(x)=R^{N} \backslash B_{\varepsilon}(x)$ and the symbol P.V. stands for the Cauchy principal value and $C(N, s)$ is a dimensional constant that depends on $N, s$, precisely given by

$$
C(N, s):=\left(\int_{R^{N}} \frac{1-\cos \left(\varsigma_{1}\right)}{|\varsigma|^{N+2 s}} d \varsigma\right)^{-1} .
$$

Fractional and nonlocal operators have recently been studied; see, for instance, [1, 2, 3, 4, 5, 6, 7, 8, 9, $10,11,12,13,14,15,16,17,18,19,20,21]$ and the references therein. These types of operators arise in a quite natural way in many different physical situations such as continuum mechanics, phase transition phenomena, population dynamics, minimal surfaces and game theory, as they are the typical outcomes of the stochastic stabilisation of the Levy processes (for more study, see [22, 23, 24] and the references therein). For the basic properties of fractional Sobolev spaces, we refer the readers to [14, 25]. Also, we know that the topological methods for fractional problems have been first set in [18, 19]. Recently, by using the mountain pass lemma, Ghoussoub and Shakerian [26] established the existence of a nontrivial weak solution to the problem

$$
(-\Delta)^{s} u-\gamma \frac{u}{|x|^{2 s}}=|u|^{2_{s}^{*}-2} u+\frac{|u|^{2_{s}^{*}(\alpha)-2} u}{|x|^{\alpha}}, \quad u>0, \quad \text { in } \mathbb{R}^{N} .
$$

Moreover, Yang and $\mathrm{Wu}$ [27] showed the existence of nontrivial solutions for doubly critical nonlocal elliptic problems in $\mathbb{R}^{N}$. For other recent results in fractional and nonlocal operators, the reader is referred to, for example, $[28,29,30,31]$ and the references therein.

In [32], Chen considered the following doubly critical problem involving the fractional Laplacian

$$
(-\Delta)^{s} u-\gamma \frac{u}{|x|^{2 s}}=\frac{|u|^{2_{s}^{*}(\alpha)-2} u}{|x|^{\alpha}}+\frac{|u|^{2_{s}^{*}(\beta)-2} u}{|x|^{\beta}}, \quad u>0, \quad \text { in } \mathbb{R}^{n},
$$

where $s \in(0,1), 0<\alpha, \beta<2 s<n$ with $\alpha \neq \beta, \gamma<\gamma_{H}$. Applying the mountain pass lemma and a concentration compactness principle, Chen proved the existence of positive solutions to (1.2). For other recent results for related problems, one can see [8, 33, 34].

In this paper, motivated by $[35,36]$, we study the multiplicity of nontrivial solutions for problem $(1.1)$. Now, we state our main result.

Theorem 1.1. Assume that $f(x, u)$ is measurable in $x$ and continuously differentiable in $u, f(x, 0)=0$ for every $x \in \mathbb{R}^{N}$, there exist $l \in\left(2,2_{s}^{*}\right)$ and constants $\mu_{1} \in\left(\frac{1}{2_{s}^{*}(\alpha)-1}, 1\right), \mu_{2} \in\left(2,2_{s}^{*}(\alpha)\right), 0<\mu_{3}<\mu_{4}$ such that, for any $u \in H_{0}^{s}\left(\mathbb{R}^{N}\right)$,

$$
\begin{aligned}
\mu_{3} \int_{\mathbb{R}^{N}} g(x)|u|^{l} d x & \leq \mu_{2} \int_{R^{N}} F(x, u) d x \leq \int_{R^{N}} f(x, u) u d x \\
& \leq \mu_{1} \int_{R^{N}} f_{u}(x, u) u^{2} d x \leq \mu_{4} \int_{\mathbb{R}^{N}} g(x)|u|^{l} d x
\end{aligned}
$$

where $F(x, u)=\int_{0}^{u} f(x, s) d s$ and $g(x)>0, g \in L^{\infty}\left(\mathbb{R}^{N}\right)$ and there exists $\rho_{0}>0$ such that suppg $\subset B_{\rho_{0}}(0)$, where $B_{\rho_{0}}(x)=\left\{y \in \mathbb{R}^{N}:|y-x|<\rho_{0}\right\}$. Then, there exists $\lambda^{*}=\lambda^{*}(N, s, \gamma, \alpha, l)$, such that for every 
$\lambda>\lambda^{*}$, there exist three different nontrivial weak solutions of problem (1.1). Moreover, these solutions are one positive, one negative and the other one sign-changing.

\section{PRELIMINARIES AND PROOF OF THE MAIN RESUlT}

In this paper, by employing Ekeland's variational principle and a similar argument in [37], we investigate the existence and multiplicity of solutions for critical nonlocal fractional Hardy elliptic equation 1.1. In order to prove our main results, we need need to present some preliminaries of the variational framework, definitions and lemmas which will play an important role to solve problem (1.1).

We first give some useful notations and basic results of fractional Sobolev space that will be used in proof of the main results. Let $0<s<1<p<\infty$ be real numbers. The fractional Sobolev space $W^{s, p}\left(\mathbb{R}^{N}\right)$ is defined by

$$
W^{s, p}\left(\mathbb{R}^{N}\right)=\left\{u \in L^{p}\left(\mathbb{R}^{N}\right): \iint_{\mathbb{R}^{2 N}} \frac{|u(x)-u(y)|^{p}}{|x-y|^{N+p s}} d x d y\right\},
$$

equipped with the norm

$$
\|u\|_{W^{s, p}\left(\mathbb{R}^{N}\right)}=\left(\|u\|_{L^{p}\left(\mathbb{R}^{N}\right)}^{p}+\iint_{\mathbb{R}^{2 N}} \frac{|u(x)-u(y)|^{p}}{|x-y|^{N+p s}} d x d y\right)^{\frac{1}{p}} .
$$

We know that, if $p=2$, then $W^{s, 2}\left(\mathbb{R}^{N}\right):=H^{s}\left(\mathbb{R}^{N}\right)$. Also, $H^{s}\left(\mathbb{R}^{N}\right)$ denotes the fractional Sobolev space of $g \in L^{2}\left(\mathbb{R}^{N}\right)$ such that the map $(x, y) \mapsto \frac{g(x)-g(y)}{|x-y|^{\frac{N+2 s}{2}}}$ is in $L^{2}\left(\mathbb{R}^{N} \times \mathbb{R}^{N}\right)$.

Let us consider $H^{s}\left(\mathbb{R}^{N}\right)$ with the norm

$$
\|u\|_{H^{s}\left(\mathbb{R}^{N}\right)}=\left(\|u\|_{L^{2}\left(\mathbb{R}^{N}\right)}^{2}+\iint_{\mathbb{R}^{2 N}} \frac{|u(x)-u(y)|^{2}}{|x-y|^{N+2 s}} d x d y\right)^{\frac{1}{2}} .
$$

We introduce the space $H_{0}^{s}\left(\mathbb{R}^{N}\right)$ as the completion of $C_{0}^{\infty}\left(\mathbb{R}^{N}\right)$ with respect to

$$
[u]_{s}=\left(\iint_{\mathbb{R}^{2 N}} \frac{|u(x)-u(y)|^{2}}{|x-y|^{N+2 s}} d x d y\right)^{\frac{1}{2}} .
$$

We know that $\left(H_{0}^{s}\left(\mathbb{R}^{N}\right),[\cdot]_{s}\right)$ is a uniformly convex Banach space. By [38, Theorem 1], one has

$$
\|u\|_{2_{s}^{*}}^{2} \leq C_{N} \frac{s(1-s)}{N-2 s}[u]_{s}^{2}, \forall u \in H_{0}^{s}\left(\mathbb{R}^{N}\right),
$$

and by [39, Theorem 1.1], one has

$$
\gamma_{1} \int_{\mathbb{R}^{N}} \frac{|u(x)|^{2}}{|x|^{2 s}} d x \leq[u]_{s}^{2}, \forall u \in H_{0}^{s}\left(\mathbb{R}^{N}\right),
$$

where

$$
\gamma_{1}:=2 \pi^{\frac{N}{2}} \frac{\Gamma\left(\frac{N+2 s}{4}\right)^{2}|\Gamma(-s)|}{\Gamma\left(\frac{N-2 s}{4}\right)^{2} \Gamma\left(\frac{N+2 s}{2}\right)} .
$$

Moreover, the constant $\gamma_{1}$ is optimal. If $\gamma<\gamma_{1}$, it follows from the Hardy inequality (2.2) that

$$
\|u\|:=\left(\iint_{\mathbb{R}^{2 N}} \frac{|u(x)-u(y)|^{2}}{|x-y|^{N+2 s}} d x d y-\gamma \int_{\mathbb{R}^{N}} \frac{|u(x)|^{2}}{|x|^{2 s}} d x\right)^{\frac{1}{2}}
$$


is well defined on $H_{0}^{S}\left(\mathbb{R}^{N}\right)$. Since

$$
\left(1-\frac{\lambda_{+}}{\gamma_{1}}\right)[u]_{s}^{2} \leq\|u\|^{2} \leq\left(1+\frac{\lambda_{-}}{\gamma_{1}}\right)[u]_{s}^{2}, \forall u \in H_{0}^{s}\left(\mathbb{R}^{N}\right)
$$

where $\lambda_{+}=\max \{\gamma, 0\}$ and $\lambda_{-}=\max \{-\gamma, 0\}$, then $\|u\|$ is comparable to $[u]_{s}$. Thus, fractional Sobolev embedding $H_{0}^{s}\left(\mathbb{R}^{N}\right) \hookrightarrow L^{2_{s}^{*}}\left(\mathbb{R}^{N}\right)$ and fractional Hardy embedding $H_{0}^{s}\left(\mathbb{R}^{N}\right) \hookrightarrow L^{2}\left(\mathbb{R}^{N},|x|^{-2 s}\right)$ are continuous, but they are not compact. Combining the Hardy inequality and the Sobolev inequality, we obtain the Hardy-Sobolev inequality. Indeed, let $\alpha \in[0,2 s)$ be a real number. Then $H_{0}^{s}\left(\mathbb{R}^{N}\right)$ is continuously embedded in the weighted space $L^{2_{s}^{*}(\alpha)}\left(\mathbb{R}^{N},|x|^{-\alpha}\right)$. Here, taking the smallest constant associated to this embedding, we let

$$
S(N, s, \gamma, \alpha)=\inf _{u \in H_{0}^{s}\left(\mathbb{R}^{N}\right) \backslash\{0\}} \frac{\iint_{\mathbb{R}^{2 N}} \frac{|u(x)-u(y)|^{2}}{|x-y|^{N+2 s}} d x d y-\gamma \int_{\mathbb{R}^{N}} \frac{|u(x)|^{2}}{|x|^{2 s}} d x}{\left(\int_{\mathbb{R}^{N}}|u(x)|^{*}|x|^{-\alpha} d x\right)^{\frac{2}{2_{s}^{*}(\alpha)}}} .
$$

By [25, Proposition 3.6], we have $[u]_{s}=\left\|(-\triangle)^{\frac{s}{2}} u\right\|_{L^{2}\left(\mathbb{R}^{N}\right)}$ for any $u \in H^{s}\left(\mathbb{R}^{N}\right)$, i.e.

$$
\iint_{\mathbb{R}^{2 N}} \frac{|u(x)-u(y)|^{2}}{|x-y|^{N+2 s}} d x d y=\int_{\mathbb{R}^{N}}\left|(-\triangle)^{\frac{s}{2}} u(x)\right|^{2} d x .
$$

So,

$$
\left.\iint_{\mathbb{R}^{2 N}} \frac{(u(x)-u(y))(v(x)-v(y))}{|x-y|^{N+2 s}} d x d y=\int_{\mathbb{R}^{N}}(-\triangle)^{\frac{s}{2}} u(x) \cdot-\triangle\right)^{\frac{s}{2}} v(x) d x,
$$

for any $u, v \in H^{s}\left(\mathbb{R}^{N}\right)$.

Lemma 2.1. Assume that $0<s<1$.

(i) (The fractional Hardy inequality [40]) For all $u \in H^{s}\left(\mathbb{R}^{N}\right)$, we have

$$
\gamma_{H} \int_{\mathbb{R}^{N}} \frac{|u|^{2}}{|x|^{2 s}} d x \leq \int_{\mathbb{R}^{N}}\left|(-\Delta)^{s / 2} u\right|^{2} d x
$$

where $\gamma_{H}=4^{s} \frac{\Gamma^{2}\left(\frac{N+2 s}{4}\right)}{\Gamma^{2}\left(\frac{N-2 s}{4}\right)}$ is the best constant in the above inequality on $\mathbb{R}^{N}$.

(ii) (The fractional Hardy-Sobolev inequality [26]) Assume $0 \leq \alpha \leq 2 s<N$. Then, there exist positive constants $c$ and $C$, such that for all $u \in H^{S}\left(\mathbb{R}^{N}\right)$,

$$
\left(\int_{\mathbb{R}^{N}} \frac{|u|^{2_{s}^{*}(\alpha)}}{|x|^{\alpha}} d x\right)^{\frac{2}{2_{s}^{*}(\alpha)}} \leq c \int_{\mathbb{R}^{N}}\left|(-\Delta)^{s / 2} u\right|^{2} d x:=c[u]_{s}^{2} .
$$

Moreover, if $\gamma<\gamma_{H}$, then

$$
\left(\int_{\mathbb{R}^{N}} \frac{|u|^{2_{s}^{*}(\alpha)}}{|x|^{\alpha}} d x\right)^{\frac{2}{2_{s}^{*}(\alpha)}} \leq C_{\alpha}\left(\int_{\mathbb{R}^{N}}\left|(-\Delta)^{s / 2} u\right|^{2} d x-\gamma \int_{\mathbb{R}^{N}} \frac{|u|^{2}}{|x|^{2 s}} d x\right)
$$

for all $u \in H^{s}\left(\mathbb{R}^{N}\right)$.

We say that $u \in H_{0}^{s}\left(\mathbb{R}^{N}\right)$ is a weak solution of problem (1.1) if

$$
\begin{array}{r}
\iint_{\mathbb{R}^{2 N}} \frac{u(x)-u(y)}{|x-y|^{N+2 s}}(\varphi(x)-\varphi(y)) d x d y-\gamma \int_{\mathbb{R}^{N}} \frac{u(x)}{|x|^{2 s}} \varphi(x) d x \\
=\int_{R^{N}} \frac{|u|^{2 *}(\alpha)-2}{|x|^{\alpha}} u(x) \varphi(x) d x-\lambda \int_{\mathbb{R}^{N}} f(x, u) \varphi(x) d x
\end{array}
$$


for any $\varphi \in H_{0}^{s}\left(\mathbb{R}^{N}\right)$. The energy functional $J: H_{0}^{s}\left(\mathbb{R}^{N}\right) \rightarrow \mathbb{R}$, which is defined by the formula

$$
\begin{aligned}
J(u)= & \frac{1}{2}\left(\iint_{\mathbb{R}^{2 N}} \frac{|u(x)-u(y)|^{2}}{|x-y|^{N+2 s}} d x d y-\gamma \int_{\mathbb{R}^{N}} \frac{|u(x)|^{2}}{|x|^{2 s}} d x\right) \\
& -\frac{1}{2_{s}^{*}(\alpha)} \int_{\mathbb{R}^{N}} \frac{|u|^{2}(\alpha)}{|x|^{\alpha}} d x-\lambda \int_{\mathbb{R}^{N}} F(x, u) d x .
\end{aligned}
$$

So a solution of (1.1) is a nontrivial critical point of $J$. From supp $g \subset B_{\rho_{0}}(0)$, the Hölder inequality and (2.10), we can get

$$
\begin{aligned}
\int_{\mathbb{R}^{N}} g(x)|u(x)|^{l} d x & \leq\|g\|_{\infty}\left(\int_{B_{\rho_{0}}(0)} 1 d x\right)^{\frac{2_{s}^{*}-l}{2_{s}^{*}}}\left(\int_{B_{\rho_{0}}(0)}|u(x)|^{2_{s}^{*}} d x\right)^{\frac{l}{2_{s}^{*}}} \\
& =\|f\|_{\infty}\left(\frac{\pi^{\frac{N}{2}}}{\Gamma\left(\frac{N}{2}+1\right)} \rho_{0}^{N}\right)^{\frac{2_{s}^{*}-l}{2_{s}^{*}}}\left(\int_{B_{\rho_{0}}(0)}|u(x)|^{2_{s}^{*}} d x\right)^{\frac{l}{2_{s}^{*}}} \\
& \leq\|g\|_{\infty}\left(\frac{\pi^{\frac{N}{2}}}{\Gamma\left(\frac{N}{2}+1\right)} \rho_{0}^{N}\right)^{\frac{2_{s}^{*}-l}{2_{s}^{*}}} C_{0}^{\frac{2_{s}^{*}}{2}}\|u\|^{l} .
\end{aligned}
$$

Recall that a sequence $\left\{u_{n}\right\}_{n \in \mathbb{N}}$ is a (PS $)_{c}$ sequence for functional $J$ if $J\left(u_{n}\right) \rightarrow c$ and $J^{\prime}\left(u_{n}\right) \rightarrow 0$. If any $(\mathrm{PS})_{c}$ sequence $\left\{u_{n}\right\}_{n \in \mathbb{N}}$ has a convergent subsequence, we say that $J$ satisfies the (PS) $)_{c}$ condition. Set

$$
\begin{aligned}
M_{1}:= & \left\{u \in H_{0}^{s}\left(\mathbb{R}^{N}\right): \int_{R^{N}} u_{+}>0, \iint_{\mathbb{R}^{2 N}} \frac{\left|u_{+}(x)-u_{+}(y)\right|^{2}}{|x-y|^{N+2 s}} d x d y\right. \\
& \left.-\gamma \int_{\mathbb{R}^{N}} \frac{\left|u_{+}(x)\right|^{2}}{|x|^{2 s}} d x-\int_{\mathbb{R}^{N}} \frac{\left|u_{+}\right|^{2_{s}^{*}(\alpha)}}{|x|^{\alpha}} d x=\lambda \int_{\mathbb{R}^{N}} f(x, u) u_{+} d x\right\}, \\
M_{2}:= & \left\{u \in H_{0}^{s}\left(\mathbb{R}^{N}\right): \int_{R^{N}} u_{-}>0, \iint_{\mathbb{R}^{2 N}} \frac{\left|u_{-}(x)-u_{-}(y)\right|^{2}}{|x-y|^{N+2 s}} d x d y\right. \\
& \left.-\gamma \int_{\mathbb{R}^{N}} \frac{\left|u_{-}(x)\right|^{2}}{|x|^{2 s}} d x-\int_{R^{N}} \frac{\left|u_{-}\right|_{s}^{*}(\alpha)}{|x|^{\alpha}} d x=-\lambda \int_{\mathbb{R}^{N}} f(x, u) u_{-} d x\right\},
\end{aligned}
$$

$M_{3}=M_{1} \cap M_{2}$,

$$
K_{1}:=\left\{u \in M_{1} ; u \geq 0\right\}, \quad K_{2}:=\left\{u \in M_{2} ; u \leq 0\right\}, \quad K_{3}:=M_{3},
$$

where $u_{+}:=\max \{u, 0\}$ and $u_{-}:=\max \{-u, 0\}$.

Lemma 2.2. Under the assumptions of Theorem 1.1, for every $u_{0} \in H_{0}^{s}\left(\mathbb{R}^{N}\right), u_{0}>0\left(u_{0}<0\right)$, there exists $t_{\lambda}>0$ such that $t_{\lambda} u_{0} \in M_{1}\left(\in M_{2}\right)$. Furthermore, $\lim _{\lambda \rightarrow \infty} t_{\lambda}=0$.

Proof. For $u \in H_{0}^{s}\left(\mathbb{R}^{N}\right)$, we define the functional

$$
\chi_{1}(u):=\iint_{\mathbb{R}^{2 N}} \frac{|u(x)-u(y)|^{2}}{|x-y|^{N+2 s}} d x d y-\gamma \int_{\mathbb{R}^{N}} \frac{|u(x)|^{2}}{|x|^{2 s}} d x-\int_{\mathbb{R}^{N}} \frac{|u|^{2_{s}^{*}(\alpha)}}{|x|^{\alpha}} d x-\lambda \int_{\mathbb{R}^{N}} f(x, u) u d x .
$$


Given $u_{0}>0$, we claim that $\chi_{1}\left(t_{\lambda} u_{0}\right)=0$ for some $t_{\lambda}>0$. To this end, we find from (1.3) and (2.12) that

$$
\begin{aligned}
\chi_{1}\left(t u_{0}\right)= & t^{2}\left[\iint_{\mathbb{R}^{2 N}} \frac{\left|u_{0}(x)-u_{0}(y)\right|^{2}}{|x-y|^{N+2 s}} d x d y-\gamma \int_{\mathbb{R}^{N}} \frac{\left|u_{0}(x)\right|^{2}}{|x|^{2 s}} d x\right] \\
& -t^{2_{s}^{*}(\alpha)} \int_{\mathbb{R}^{N}} \frac{\left|u_{0}\right|^{2}(\alpha)}{|x|^{\alpha}} d x-\lambda \int_{\mathbb{R}^{N}} f\left(x, t u_{0}\right) t u_{0} d x \\
\geq & t^{2}\left[\iint_{\mathbb{R}^{2 N}} \frac{\left|u_{0}(x)-u_{0}(y)\right|^{2}}{|x-y|^{N+2 s}} d x d y-\gamma \int_{\mathbb{R}^{N}} \frac{\left|u_{0}(x)\right|^{2}}{|x|^{2 s}} d x\right] \\
& -t^{2_{s}^{*}(\alpha)} \int_{\mathbb{R}^{N}} \frac{\left|u_{0}\right|^{2_{s}^{*}(\alpha)}}{|x|^{\alpha}} d x-\lambda t^{l} \mu_{4} \int_{\mathbb{R}^{N}} g(x)\left|u_{0}\right|^{l} d x \\
\geq & A t^{2}-B t^{2_{s}^{*}(\alpha)}-\lambda \mu_{4} t^{l} C,
\end{aligned}
$$

where $A=\iint_{\mathbb{R}^{2 N}} \frac{\left|u_{0}(x)-u_{0}(y)\right|^{2}}{|x-y|^{N+2 s}} d x d y-\gamma \int_{\mathbb{R}^{N}} \frac{\left|u_{0}(x)\right|^{2}}{|x|^{2 s}} d x, B=\int_{\mathbb{R}^{N}} \frac{\left|u_{0}\right|^{2 *}(\alpha)}{|x|^{\alpha}} d x$ and

$$
C=\|g\|_{\infty}\left(\frac{\pi^{\frac{N}{2}}}{\Gamma\left(\frac{N}{2}+1\right)} \rho_{0}^{N}\right)^{\frac{2_{s}^{*}-l}{2 s}} C_{0}^{\frac{2_{s}^{*}}{2}}\left\|u_{0}\right\|^{l} .
$$

Similarly we can get

$$
\chi_{1}\left(t u_{0}\right) \leq A t^{2}-B t^{2_{s}^{*}(\alpha)}-\lambda \mu_{3} t^{l} C .
$$

Since $2<l<2_{s}^{*}(\alpha)$, Bolzano's theorem yields that there exists $t=t_{\lambda}$ such that $\varphi_{1}\left(t_{\lambda} u\right)=0$. Also, from $\chi_{1}\left(t u_{0}\right) \leq A t^{2}-\lambda \mu_{3} C t^{l}$, we choose $t_{1}$ such that $A t_{1}^{2}-\lambda \mu_{3} C t_{1}^{l}=0$. So, $t_{1}=\left(\frac{A}{\lambda \mu_{3} C}\right)^{\frac{1}{l-2}}$. Choosing $t_{\lambda} \in\left[0, t_{1}\right]$, we obtain the desired conclusion immediately.

Corollary 2.3. By Lemma 2.2, for any $u_{0}, u_{1} \in H_{0}^{s}\left(\mathbb{R}^{N}\right), u_{0}>0$ and $u_{1}<0$ with disjoint supports, there exists $t_{1 \lambda}, t_{2 \lambda}>0$ such that $t_{\lambda}^{*} u_{0}+t_{\lambda}^{* *} u_{1} \in M_{3}$. Moreover $t_{\lambda}^{*}, t_{\lambda}^{* *} \rightarrow 0$ as $\lambda \rightarrow \infty$.

Lemma 2.4. There exist $\eta_{1}$ and $\eta_{2}>0$ such that, for every $u \in K_{i}, i=1,2,3$,

$$
\|u\|^{2}=\int_{\mathbb{R}^{N}} \frac{|u|^{2_{s}^{*}(\alpha)}}{|x|^{\alpha}} d x+\lambda \int_{\mathbb{R}^{N}} f(x, u) u d x \leq \eta_{1} J(u) \leq \eta_{2}\|u\|^{2} .
$$

Proof. Since $u \in K_{i}$, one sees that the equality is clear. In view of (1.3), one can get

$$
\begin{aligned}
J(u)= & \frac{1}{2}\left(\iint_{\mathbb{R}^{2 N}} \frac{|u(x)-u(y)|^{2}}{|x-y|^{N+2 s}} d x d y-\gamma \int_{\mathbb{R}^{N}} \frac{|u(x)|^{2}}{|x|^{2 s}} d x\right) \\
& -\frac{1}{2_{s}^{*}(\alpha)} \int_{\mathbb{R}^{N}} \frac{|u|^{2_{s}^{*}(\alpha)}}{|x|^{\alpha}} d x-\lambda \int_{\mathbb{R}^{N}} F(x, u) d x \\
= & \frac{1}{2}\left(\int_{\mathbb{R}^{N}} \frac{|u|^{2_{s}^{*}(\alpha)}}{|x|^{\alpha}} d x+\lambda \int_{\mathbb{R}^{N}} f(x, u) u d x\right)-\frac{1}{2_{s}^{*}(\alpha)} \int_{\mathbb{R}^{N}} \frac{|u|^{2_{s}^{*}(\alpha)}}{|x|^{\alpha}} d x-\lambda \int_{\mathbb{R}^{N}} F(x, u) d x \\
= & \left(\frac{1}{2}-\frac{1}{2_{s}^{*}(\alpha)}\right) \int_{R^{N}} \frac{|u|^{2_{s}^{*}(\alpha)}}{|x|^{\alpha}}+\frac{\lambda}{2} \int_{\mathbb{R}^{N}} f(x, u) u d x-\lambda \int_{\mathbb{R}^{N}} F(x, u) d x \\
\geq & \left(\frac{1}{2}-\frac{1}{2_{s}^{*}(\alpha)}\right) \int_{\mathbb{R}^{N}} \frac{|u|^{2_{s}^{*}(\alpha)}}{|x|^{\alpha}}+\left(\frac{1}{2}-\frac{1}{\mu_{2}}\right) \lambda \int_{\mathbb{R}^{N}} f(x, u) u d x .
\end{aligned}
$$

Since $2<\mu_{2}<2_{s}^{*}(\alpha)$, one finds that the first inequality hold. By $F(x, u) \geq 0$, the last inequality is clear. 
Lemma 2.5. There exists $C>0$ such that

$$
\begin{aligned}
& \left\|u_{+}\right\| \geq C, \quad \forall u \in K_{1}, \\
& \left\|u_{-}\right\| \geq C, \quad \forall u \in K_{2}, \\
& \left\|u_{+}\right\|,\left\|u_{-}\right\| \geq C, \quad \forall u \in K_{3} .
\end{aligned}
$$

Proof. By the definition of $K_{i}(i=1,2,3), 2<l<2_{s}^{*}(\alpha),(1.3),(2.10)$ and (2.12), one can get

$$
\begin{aligned}
\left\|u_{ \pm}\right\|^{2} & =\lambda \int_{R^{N}} f(x, u) u_{ \pm} d x+\int_{\mathbb{R}^{N}} \frac{\left|u_{ \pm}\right|^{2_{s}^{*}}(\alpha)}{|x|^{\alpha}} d x \\
& \leq c_{1} \int_{\mathbb{R}^{N}} g(x)\left|u_{ \pm}\right|^{l} d x+\int_{\mathbb{R}^{N}} \frac{\left|u_{ \pm}\right|^{2_{s}^{*}(\alpha)}}{|x|^{\alpha}} d x \\
& \leq c_{1}\|g\|_{\infty}\left(\frac{\pi^{\frac{N}{2}}}{\Gamma\left(\frac{N}{2}+1\right)} \rho_{0}^{N}\right)^{\frac{2_{s}^{*}-l}{2_{s}^{*}}} C_{0}^{\frac{2_{s}^{*}}{2}}\left\|u_{ \pm}\right\|^{l}+C_{\alpha}^{\frac{2_{s}^{*}(\alpha)}{2}}\left\|u_{ \pm}\right\|^{2_{s}^{*}(\alpha)}
\end{aligned}
$$

where $c_{1}$ is a positive constant. Therefore, we have the conclusion.

Lemma 2.6. There exists $\Theta>0$ such that $J(u) \geq \Theta\|u\|^{2}, \forall u \in H_{0}^{s}\left(\mathbb{R}^{N}\right)$ if $\|u\|$ is small enough.

Proof. In view of (1.3), (2.10) and (2.12), we get

$$
\begin{aligned}
J(u)= & \frac{1}{2}\left(\iint_{\mathbb{R}^{2 N}} \frac{|u(x)-u(y)|^{2}}{|x-y|^{N+2 s}} d x d y-\gamma \int_{\mathbb{R}^{N}} \frac{|u(x)|^{2}}{|x|^{2 s}} d x\right) \\
& -\frac{1}{2_{s}^{*}(\alpha)} \int_{\mathbb{R}^{N}} \frac{|u|^{2_{s}^{*}(\alpha)}}{|x|^{\alpha}} d x-\lambda \int_{\mathbb{R}^{N}} F(x, u) d x \\
\geq & \frac{1}{2}\|u\|^{2}-\frac{1}{2_{s}^{*}(\alpha)} \int_{\mathbb{R}^{N}} \frac{|u|^{2_{s}^{*}(\alpha)}}{|x|^{\alpha}} d x-c_{2} \int_{\mathbb{R}^{N}} g(x)|u|^{l} d x \\
\geq & \frac{1}{2}\|u\|^{2}-\frac{1}{2_{s}^{*}(\alpha)} C_{\alpha}^{\frac{2_{s}^{*}(\alpha)}{2}}\|u\|^{2_{s}^{*}(\alpha)}-c_{2}\|g\|_{\infty}\left(\frac{\pi^{\frac{N}{2}}}{\Gamma\left(\frac{N}{2}+1\right)} \rho_{0}^{N}\right)^{\frac{2_{s}^{*}-l}{2_{s}^{*}}} C_{0}^{\frac{2_{s}^{*}}{2}}\|u\|^{l} \\
= & \|u\|^{2}\left(\frac{1}{2}-\frac{1}{2_{s}^{*}(\alpha)} C_{\alpha}^{\frac{2_{s}^{*}(\alpha)}{2}}\|u\|^{2_{s}^{*}(\alpha)-2}-c_{2}\|g\|_{\infty}\left(\frac{\pi^{\frac{N}{2}}}{\Gamma\left(\frac{N}{2}+1\right)} \rho_{0}^{N}\right)^{\frac{2_{s}^{*}-l}{2_{s}^{*}}} C_{0}^{\frac{2_{s}^{*}}{2}}\|u\|^{l-2}\right) .
\end{aligned}
$$

Since $2<l<2_{s}^{*}(\alpha)$, we find that $J(u) \geq C\|u\|^{2}$ if $\|u\|$ is small enough.

Lemma 2.7. $M_{i}$ is a $C^{1}$ sub-manifold with co-dimension $1(i=1,2), 2(i=3)$. The sets $K_{i}(i=1,2,3)$ are complete. Moreover, for every $u \in M_{i}$,

$$
\begin{gathered}
T_{u} H_{0}^{s}\left(\mathbb{R}^{\mathbb{N}}\right)=T_{u} M_{1} \oplus \operatorname{span}\left\{u_{+}\right\}, \\
T_{u} H_{0}^{s}\left(\mathbb{R}^{\mathbb{N}}\right)=T_{u} M_{2} \oplus \operatorname{span}\left\{u_{-}\right\}, \\
T_{u} H_{0}^{s}\left(\mathbb{R}^{\mathbb{N}}\right)=T_{u} M_{3} \oplus \operatorname{span}\left\{u_{+}, u_{-}\right\},
\end{gathered}
$$

where $T_{u} M$ is the tangent space at $u$ of the Banach manifold M. Moreover, the projection onto the first component in this decomposition is uniformly continuous on bounded sets of $M_{i}$. 
Proof. Similar to the method in [35], we set

$$
\begin{aligned}
& \bar{M}_{1}:=\left\{u \in H_{0}^{s}\left(\mathbb{R}^{\mathbb{N}}\right): \int_{\mathbb{R}^{N}} u_{+} d x>0\right\} ; \\
& \bar{M}_{2}:=\left\{u \in H_{0}^{s}\left(\mathbb{R}^{\mathbb{N}}\right): \int_{\mathbb{R}^{N}} u_{-} d x>0\right\} ; \\
& \bar{M}_{3}:=\bar{M}_{1} \bigcap \bar{M}_{2} .
\end{aligned}
$$

Notice that $M_{i} \subset \bar{M}_{i}$ and $\bar{M}_{i}$ is open in $H_{0}^{s}\left(\mathbb{R}^{\mathbb{N}}\right)$. We claim that $M_{i}$ is a $C^{1}$ sub-manifold of $\bar{M}_{i}$. To this end, we will construct a $C^{1}$ function $\varphi_{i}: \bar{M}_{i} \rightarrow \mathbb{R}^{d}$ with $d=1(i=1,2), d=2(i=3)$ respectively and $M_{i}$ will be the inverse image of a regular value of $\varphi_{i}$. So, we define

$$
\begin{aligned}
\varphi_{1}(u)= & \iint_{\mathbb{R}^{2 N}} \frac{\left.\left|u_{+}(x)-\right| u_{+}(y)\right|^{2}}{|x-y|^{N+2 s}} d x d y-\gamma \int_{\mathbb{R}^{N}} \frac{\left|u_{+}(x)\right|^{2}}{|x|^{2 s}} d x \\
& -\int_{\mathbb{R}^{N}} \frac{\left|u_{+}\right|^{2 *}(\alpha)}{|x|^{\alpha}} d x-\lambda \int_{\mathbb{R}^{N}} f(x, u) u_{+} d x, \quad \forall u \in \bar{M}_{1}, \\
\varphi_{2}(u)= & \iint_{\mathbb{R}^{2 N}} \frac{\left.\left|u_{-}(x)-\right| u_{-}(y)\right|^{2}}{|x-y|^{N+2 s}} d x d y-\gamma \int_{\mathbb{R}^{N}} \frac{\left|u_{-}(x)\right|^{2}}{|x|^{2 s}} d x \\
& -\int_{\mathbb{R}^{N}} \frac{\left|u_{-}\right|_{s}^{*}(\alpha)}{|x|^{\alpha}} d x-\lambda \int_{\mathbb{R}^{N}} f(x, u) u_{-} d x, \quad \forall u \in \bar{M}_{2}, \\
\varphi_{3}(u)= & \left(\varphi_{1}(u), \varphi_{2}(u)\right), \quad \forall u \in \bar{M}_{3} .
\end{aligned}
$$

Clearly, $M_{i}=\varphi_{i}^{-1}(0)$. We now claim that $\varphi_{i}$ is of class $C^{1}$ (we will prove that $\varphi_{1} \in C^{1}$, and by similar method, $\left.\varphi_{2} \in C^{1}\right)$. To this end, set

$$
\psi(u)=\int_{\mathbb{R}^{N}} f(x, u) u_{+} d x
$$

It suffices to show that $\psi \in C^{1}$,

$$
<\psi^{\prime}(u), u_{+}>=\int_{\mathbb{R}^{N}}\left[f_{u}(x, u) u_{+}^{2}+f(x, u) u_{+}\right] d x, \forall u \in H_{0}^{s}\left(\mathbb{R}^{\mathbb{N}}\right) .
$$

First we prove the existence of the Gateaux derivative of $\psi$. Since $f(x, u)$ is continuously differentiable in $u$, one finds from the mean value theorem that there exists $0<\theta<1$ and $0<|\mu|<1$ such that

$$
\frac{f\left(x, u(x)+\mu u_{+}(x)\right)-f(x, u(x))}{\mu u_{+}(x)}=f_{u}\left(x, u(x)+\theta \mu u_{+}(x)\right) .
$$

Using (1.3), (2.13) and the Lebesgue's dominated convergence theorem, we have

$$
\begin{aligned}
<\psi^{\prime}(u), u_{+}> & =\lim _{\mu \rightarrow 0^{+}} \frac{\psi\left(u+\mu u_{+}\right)-\psi(u)}{\mu} \\
& =\lim _{\mu \rightarrow 0^{+}} \int_{\mathbb{R}^{N}} \frac{f\left(x, u(x)+\mu u_{+}(x)\right)\left(u_{+}(x)+\mu u_{+}(x)\right)-f(x, u(x)) u_{+}(x)}{\mu} d x \\
& =\lim _{\mu \rightarrow 0^{+}} \int_{\mathbb{R}^{N}}\left[\frac{f\left(x, u(x)+\mu u_{+}(x)\right)-f(x, u(x))}{\mu} u_{+}(x)\right. \\
& \left.\quad+f\left(x, u(x)+\mu u_{+}(x)\right) u_{+}(x)\right] d x \\
= & \int_{\mathbb{R}^{N}}\left[f_{u}(x, u(x)) u_{+}^{2}(x)+f(x, u(x)) u_{+}(x)\right] d x .
\end{aligned}
$$


Hence, we can easily get

$$
\begin{aligned}
<\varphi_{1}^{\prime}(u), u_{+}>= & 2 \iint_{\mathbb{R}^{2 N}} \frac{\left|u_{+}(x)-u_{+}(y)\right|^{2}}{|x-y|^{N+2 s}}-2 \gamma \int_{R^{N}} \frac{\left|u_{+}\right|^{2}}{|x|^{2 s}} \\
& -2_{s}^{*}(\alpha) \int_{\mathbb{R}^{N}} \frac{\left|u_{+}\right|^{2^{*}}(\alpha)}{|x|^{\alpha}}-\lambda \int_{\mathbb{R}^{N}}\left[f_{u}(x, u) u_{+}^{2}+f(x, u) u_{+}\right] d x .
\end{aligned}
$$

Next, we show that $\psi^{\prime}(\cdot): H_{0}^{s}\left(\mathbb{R}^{\mathbb{N}}\right) \rightarrow\left(H_{0}^{s}\left(\mathbb{R}^{\mathbb{N}}\right)\right)^{*}$ is continuous. Assume that $u_{n} \rightarrow u$ in $H_{0}^{s}\left(\mathbb{R}^{\mathbb{N}}\right)$. From (1.3) and continuity $f$ and $f_{u}$, one has

$$
\begin{aligned}
\left\|\psi^{\prime}\left(u_{n}\right)-\psi^{\prime}(u)\right\|_{H_{0}^{s}\left(\mathbb{R}^{\mathbb{N}}\right)}= & \sup _{\left\|u_{+}\right\| \leq 1} \int_{\mathbb{R}^{N}} \mid\left(f_{u}\left(x, u_{n}(x)\right)-f_{u}(x, u(x))\right) u_{+}^{2}(x) \\
& +\left(f\left(x, u_{n}(x)\right)-f(x, u(x))\right) u_{+}(x) d x \mid \\
= & \sup _{\left\|u_{+}\right\| \leq 1}\left[\int_{\mathbb{R}^{N}}\left|f_{u}\left(x, u_{n}(x)\right)-f_{u}(x, u(x))\right|\left|u_{+}^{2}(x)\right|\right. \\
& \left.+\left|f\left(x, u_{n}(x)\right)-f(x, u(x)) \| u_{+}(x)\right| d x\right] \rightarrow 0 \text { as } n \rightarrow \infty .
\end{aligned}
$$

Hence $\left\|\psi^{\prime}\left(u_{n}\right)-\psi^{\prime}(u)\right\|_{H_{0}^{s}\left(\mathbb{R}^{\mathbb{N}}\right)} \rightarrow 0$. This shows that $\psi^{\prime}$ is continuous and so $\varphi_{1} \in C^{1}$. Thus, we only need to prove that 0 is a regular value for $\varphi_{i}$. Note that $2<2_{s}^{*}(\alpha)$ and $\frac{1}{2_{s}^{*}(\alpha)-1}<\mu_{1}<1$. Then for $u \in M_{1}$, we conclude from (1.3) that

$$
\begin{aligned}
<\varphi_{1}^{\prime}(u), u_{+}>= & 2 \iint_{\mathbb{R}^{2 N}} \frac{\left|u_{+}(x)-\right| u_{+}(y) \mid}{|x-y|^{N+2 s}}\left(u_{+}(x)-u_{+}(y)\right) d x d y-2 \gamma \int_{\mathbb{R}^{N}} \frac{\left|u_{+}(x)\right|}{|x|^{2 s}} u_{+}(x) d x \\
& -2_{s}^{*}(\alpha) \int_{\mathbb{R}^{N}} \frac{\left|u_{+}\right|^{2^{*}(\alpha)-2}}{|x|^{\alpha}} u_{+}(x) d x-\lambda\left(\int_{\mathbb{R}^{N}} f(x, u) u_{+}+f_{u}(x, u) u_{+}^{2}\right) d x . \\
\leq & 2 \|\left. u\right|^{2}-2_{s}^{*}(\alpha) \int_{\mathbb{R}^{N}} \frac{\left|u_{+}\right|^{2_{s}^{*}(\alpha)-2}}{|x|^{\alpha}} u_{+}(x) d x-\lambda\left(\int_{\mathbb{R}^{N}} f(x, u) u_{+}+f_{u}(x, u) u_{+}^{2}\right) d x \\
\leq & 2\left(\|\left. u\right|^{2}-\int_{\mathbb{R}^{N}} \frac{\left|u_{+}\right|_{s}^{*}(\alpha)-2}{|x|^{\alpha}} u_{+}(x) d x\right)-\lambda\left(\int_{\mathbb{R}^{N}} f(x, u) u_{+}+f_{u}(x, u) u_{+}^{2}\right) d x \\
\leq & 2\left(\lambda \int_{\mathbb{R}^{N}} f(x, u) u_{+} d x\right)-\lambda \int_{\mathbb{R}^{N}} f(x, u) u_{+} d x-\lambda \int_{\mathbb{R}^{N}} f_{u}(x, u) u_{+}^{2} d x \\
\leq & \lambda \int_{\mathbb{R}^{N}} f(x, u) u_{+} d x-\lambda \int_{\mathbb{R}^{N}} f_{u}(x, u) u_{+}^{2} d x \\
\leq & \lambda \mu_{1} \int_{\mathbb{R}^{N}} f_{u}(x, u) u_{+}^{2} d x-\lambda \int_{\mathbb{R}^{N}} f_{u}(x, u) u_{+}^{2} d x \\
\leq & \lambda \mu_{1}\left(1-\frac{1}{\mu_{1}}\right) \int_{\mathbb{R}^{N}} f_{u}(x, u) u_{+}^{2} d x \\
\leq & \lambda \mu_{4}\left(1-\frac{1}{\mu_{1}}\right) \int_{\mathbb{R}^{N}} g(x)\left|u_{+}\right|^{l} d x<0 .
\end{aligned}
$$


Therefore $M_{1}$ is a $C^{1}$ sub-manifold of $H_{0}^{s}\left(\mathbb{R}^{N}\right)$. By similar method, we have that $M_{2}$ is a $C^{1}$ sub-manifold of $H_{0}^{s}\left(\mathbb{R}^{N}\right)$. Also, for $u \in M_{3}$, we have $<\varphi_{1}^{\prime}(u), u_{-}>=<\varphi_{2}^{\prime}(u), u_{+}>=0$. Then $M_{3}$ is also a $C^{1}$ submanifold of $H_{0}^{s}\left(\mathbb{R}^{N}\right)$. The remainder of the proof can be proved by using Lemma 2.5 and Lemma 5 in [35]. So, we omit it here.

Lemma 2.8. The restricted functional $\left.J\right|_{K_{i}}$ satisfies the $(P S)_{c}$ condition for every

$$
0<c<\frac{2 s-\alpha}{2(N-\alpha)}(S(N, s, \gamma, \alpha))^{\frac{N-\alpha}{2 s-\alpha}}
$$

Proof. From [34], we have that functional $J$ satisfies the $(\mathrm{PS})_{c}$ condition for every

$$
0<c<\frac{2 s-\alpha}{2(N-\alpha)}(S(N, s, \gamma, \alpha))^{\frac{N-\alpha}{2 s-\alpha}} .
$$

Therefore, by similar method in the proof of Lemma 7 in [35], we have the desired conclusion.

We note that if $u \in K_{i}$ be a critical point of the restricted functional $\left.J\right|_{K_{i}}$, then we know from Lemma 2.8 that $u$ is also a critical point of the unrestricted functional $J$, and so a weak solution to (1.1).

We now prove Theorem 1.1 by using Ekeland's variational principle [37].

Proof of Theorem 1.1. To prove Theorem 1.1, we need to check that functional $\left.J\right|_{K_{i}}$ satisfies the hypotheses of Ekeland's variational principle [37]. The fact that $J$ is bounded below over $K_{i}$ is a direct consequence of the construction of the manifold $K_{i}$. Then, by Ekeland's variational principle, there exists $v_{k} \in K_{i}$ such that

$$
J\left(v_{k}\right) \rightarrow c_{i}:=\inf _{K_{i}} J(u),\left.J^{\prime}\right|_{K_{i}}\left(v_{k}\right) \rightarrow 0 .
$$

We have to check that if we choose $\lambda$ large, we have that $0<c<\frac{2 s-\alpha}{2(N-\alpha)}(S(N, s, \gamma, \alpha))^{\frac{N-\alpha}{2 s-\alpha}}$. This easily follows from Lemma 2.2. For instance, for $\mu_{1}$, we choose $w_{0} \geq 0$ such that

$$
\mu_{1} \leq J\left(t_{\lambda} w_{0}\right) \leq \frac{1}{2} t_{\lambda}^{2}\left\|w_{0}\right\|^{2}
$$

Hence $\mu_{1} \rightarrow 0$ as $\lambda \rightarrow 0$. Moreover, it follows from the estimate of $t_{\lambda}$ in Lemma 2.2 that $0<c<$ $\frac{2 s-\alpha}{2(N-\alpha)}(S(N, s, \gamma, \alpha))^{\frac{N-\alpha}{2 s-\alpha}}$ for $\lambda>\lambda *$. The other cases are similar. From Lemma 2.8 , it follows that $v_{k}$ has a convergent subsequence that we still call $v_{k}$. Therefore $J$ has a critical point in $K_{i},(i=1,2,3)$. By construction, one finds that one of them is positive, other is negative and the last one changes sign.

\section{Acknowledgements}

The authors would like to thank the referees for their suggestions and helpful comments which improved the presentation of the original manuscript.

\section{REFERENCES}

[1] V. Ambrosio, Ground states solutions for a non-linear equation involving a pseudo-relativistic Schrödinger operator, J. Math. Phys. 57 (2016), Article ID 051502.

[2] V. Ambrosio, H. Hajaiej, Multiple solutions for a class of nonhomogeneous fractional Schrödinger equations in $\mathbb{R}^{N}$, J. Dynam. Differential Equations 30 (2018), 1119-1143.

[3] V. Ambrosio, Periodic solutions for critical fractional equations, Calc. Var. Partial Differential Equations 57 (2018), 45.

[4] V. Ambrosio, Concentration phenomena for critical fractional Schrödinger systems, Commun. Pure Appl. Anal. 17 (2018), 2085-2123. 
[5] M. Caponi, P. Pucci, Existence theorems for entire solutions of stationary Kirchhoff fractional $p$-Laplacian equations, Ann. Mat. Pura Appl. 95 (2016), 2099-2129.

[6] S. Dipierro, M. Medina, E. Valdinoci, Fractional elliptic problems with critical growth in the whole of $\mathbb{R}^{n}$, Appunti. Scuola Normale Superiore di Pisa (Nuova Serie) [Lecture Notes. Scuola Normale Superiore di Pisa (New Series)], 15. Edizioni della Normale, Pisa, viii+152 pp (2017).

[7] P. Felmer, A. Quaas, J.Tan, Positive solutions of the nonlinear Schrödinger equation with the fractional Laplacian, Proc. Roy. Soc. Edinburgh Sect. A 142 (2012), 1237-1262.

[8] A. Fiscella, P. Pucci, On certain nonlocal Hardy-Sobolev critical elliptic Dirichlet problems, Adv. Differential Equations 21 (2016), 571-599.

[9] A. Fiscella, P. Pucci, $p$-fractional Kirchhoff equations involving critical nonlinearities, Nonlinear Anal. Real World Appl. 35 (2017), 350-378.

[10] A. Fiscella, P. Pucci, S. Saldi, Existence of entire solutions for Schrödinger-Hardy systems involving two fractional operators, Nonlinear Anal. 158 (2017), 109-131.

[11] A. Fiscella, P. Pucci and B. Zhang, p-fractional Hardy-Schrödinger-Kirchhoff systems with critical nonlinearities, Adv. Nonlinear Anal. 8 (2019), 1111-1131.

[12] X. Mingqi, G. Molica Bisci, G. Tian, B. Zhang, Infinitely many solutions for the stationary Kirchhoff problems involving the fractional $p$-Laplacian, Nonlinearity 29 (2016), 357-374.

[13] G. Molica Bisci and V. Rădulescu, Ground state solutions of scalar field fractional Schrödinger equations, Calc. Var. Partial Differential Equations 54 (2015), 2985-3008.

[14] G. Molica Bisci, V. Rădulescu, R. Servadei, Variational methods for nonlocal fractional problems, With a foreword by Jean Mawhin. Encyclopedia of Mathematics and its Applications, 162. Cambridge University Press, Cambridge, 2016. xvi+383 pp.

[15] G. Molica Bisci, D. Repovš, On doubly nonlocal fractional elliptic equations, Atti Accad. Naz. Lincei Rend. Lincei Mat. Appl. 26 (2015), 161-176.

[16] P. Pucci, M.Q. Xiang, B.L. Zhang, Multiple solutions for nonhomogeneous Schrödinger-Kirchhoff type equations involving the fractional $p$-Laplacian in $\mathbb{R}^{N}$, Calc. Var. Partial Differential Equations 54 (2015), 2785-2806.

[17] S. Secchi, Ground state solutions for nonlinear fractional Schrödinger equations in $\mathbb{R}^{N}$, J. Math. Phys. 54 (2013), Article ID 031501.

[18] R. Servadei, E. Valdinoci, Mountain pass solutions for non-local elliptic operators, J. Math. Anal. Appl. 389 (2012), 887-898.

[19] R. Servadei, E. Valdinoci, Variational methods for non-local operators of elliptic type, Discrete Contin. Dyn. Syst. 33 (2013), 2105-2137.

[20] R. Servadei, E. Valdinoci, The Brezis-Nirenberg result for the fractional Laplacian, Trans. Amer. Math. Soc. 367 (2015), 67-102.

[21] L. Wang, B. Zhang, H. Zhang, Fractional Laplacian system involving doubly critical nonlinearities in $\mathbb{R}^{N}$, Electron. J. Qual. Theory Differ. Equ. 2017 (2017), Article ID 57.

[22] D. Applebaum, Lévy processes-from probability to finance and quantum groups, Notices Amer. Math. Soc. 51 (2004), 1336-1347.

[23] L. Caffarelli, Non-local diffusions, drifts and games, Nonlinear partial differential equations, 37C52, Abel Symp., 7, Springer, Heidelberg, 2012.

[24] N. Laskin, Fractional quantum mechanics and Lévy path integrals, Phys. Lett. A 268 (2000), 298-305.

[25] E. Di Nezza, G. Palatucci, E. Valdinoci, Hitchhiker's guide to the fractional Sobolev spaces, Bull. Sci. Math. 136 (2012), 521-573.

[26] N. Ghoussoub, S. Shakerian, Borderline variational problems involving fractional Laplacians and critical singularities, Adv. Nonlinear Stud. 15 (2015), 527-555.

[27] J. Yang, F. Wu, Doubly critical problems involving fractional Laplacians in $\mathbb{R}^{N}$, Adv. Nonlinear Stud. 17 (2017), $677-690$.

[28] B. Abdellaoui, R. Bentifour, Caffarelli-Kohn-Nirenberg type inequalities of fractional order with applications, J. Funct. Anal. 272 (2017), 3998-4029. 
[29] S. Dipierro, L. Montoro, I. Peral, B. Sciunzi, Qualitative properties of positive solutions to nonlocal critical problems involving the Hardy-Leray potential, Calc. Var. Partial Differential Equations 55 (2016), Article ID 99.

[30] S. Dipierro, E. Valdinoci, A density property for fractional weighted Sobolev spaces, Atti Accad. Naz. Lincei Rend. Lincei Mat. Appl. 26 (2015), 397-422.

[31] R.L. Frank, On the uniqueness of ground states of non-local equations, J. Èqu. Dériv. Partielles (2011), 1-10.

[32] W. Chen, Fractional elliptic problems with two critical Sobolev-Hardy exponents, Electron. J. Differential Equations 2018 (2018), Article ID 22.

[33] B. Barrios, M. Medina, I. Peral, Some remarks on the solvability of non-local elliptic problems with the Hardy potential, Commun. Contemp. Math. 16 (2014), Article ID 1350046.

[34] S. Rastegarzadeh, N. Nyamoradi, Existence of positive solutions for Hardy nonlocal fractional elliptic equations involving critical nonlinearities, Topol. Meth. Nonlinear Anal. Accepted.

[35] P.L. De Nápoli, J.F. Bonder, A. Silva, Multiple solutions for the $p$-Laplace operator with critical growth, Nonlinear Anal. 71 (2009), 6283-6289.

[36] Y. Li, The existence of solutions for quasilinear elliptic problems with multiple Hardy terms, Appl. Math. Lett. 81 (2018), 7-13.

[37] I. Ekeland, On the variational principle, J. Math. Anal. Appl. 47 (1974), 324-353.

[38] V. Maz'ya, T. Shaposhnikova, On the Bourgain, Brezis, and Mironescu theorem concerning limiting embeddings of fractional Sobolev spaces, J. Funct. Anal. 195 (2002), 230-238.

[39] R.L. Frank, R. Seiringer, Non-linear ground state representations and sharp Hardy inequalities, J. Funct. Anal. 255 (2008), 3407-3430.

[40] R. L. Frank, E. H. Lieb, R. Seiringer, Hardy-Lieb-Thirring inequalities for fractional Schrödinger operators, J. Amer. Math. Soc. 21 (2008), 925-950. 